\title{
REFINED OPERATOR INEQUALITIES FOR RELATIVE OPERATOR ENTROPIES
}

\author{
SHUZHOU WANG AND ZHENHUA WANG
}

\begin{abstract}
In this paper, we investigate the relative operator entropies in the more general settings of $\mathrm{C}^{*}$-algebras, real $\mathrm{C}^{*}$-algebras and $\mathrm{JC}$-algebras. We show that all the operator inequalities on relative operator entropies still hold in these broader settings. In addition, we improve the lower and upper bounds of the relative operator $(\alpha, \beta)$-entropy established by Nikoufar in [16 and 18] which refined the bounds for the relative operator entropy obtained by Fujii and Kamei 8 , 9 .
\end{abstract}

\section{INTRODUCTION}

It is well known that entropy first appeared in thermodynamics. It plays an important role in quantum mechanics and is a fundamental notion for quantum information theory. In 21, Segal gave a mathematical formulation of the entropy of a state of a semi-finite von Neumann algebra, which included the cases of the information theory and quantum statistical mechanics. To investigate the background of Segal's definition, Nakamura and Umegaki 14 considered the operator entropy $-A \log (A)$ for positive invertible operator $A$. Later, Umegaki [23] introduced the relative operator entropy to discuss the measures of entropy and information. The concept of relative operator entropy for strictly positive operators in noncommutative information theory was initially introduced by Fujii and Kamei in [8, 9] as follows:

$$
S(A \mid B)=A^{\frac{1}{2}}\left[\log \left(A^{-\frac{1}{2}} B A^{-\frac{1}{2}}\right)\right] A^{\frac{1}{2}} .
$$

Extending the relative operator entropy, Furuta in [10] defined the generalized relative operator entropy for the strictly positive operators $A, B$ and $\alpha \in \mathbb{R}$ by setting

$$
S_{\alpha}(A \mid B)=A^{\frac{1}{2}}\left[\left(A^{-\frac{1}{2}} B A^{-\frac{1}{2}}\right)^{\alpha} \log \left(A^{-\frac{1}{2}} B A^{-\frac{1}{2}}\right)\right] A^{\frac{1}{2}} .
$$

If $\alpha=0$, then $S_{0}(A \mid B)$ precisely is the relative operator entropy. Moreover, Nikoufar [17] introduced the notion of the relative operator $(\alpha, \beta)$-entropy as follows:

$$
S_{\alpha, \beta}(A \mid B)=A^{\frac{\beta}{2}}\left[\left(A^{-\frac{\beta}{2}} B A^{-\frac{\beta}{2}}\right)^{\alpha} \log \left(A^{-\frac{\beta}{2}} B A^{-\frac{\beta}{2}}\right)\right] A^{\frac{\beta}{2}}
$$

for invertible positive operators $A, B$ and any real numbers $\alpha, \beta$. In particular, $S_{\alpha, 1}(A \mid B)=S_{\alpha}(A \mid B)$ and $S_{0,1}(A \mid B)=S(A \mid B)$.

In a different direction, Lieb [12] and Lieb and Ruskai [13] published in 1973 two articles on operator inequalities that had a significant impact on quantum mechanics

2010 Mathematics Subject Classification. Primary 47A63, 94A17, 47A56, 46L70, 47A60; Secondary 46N50, 17C65, 47L30, 81R15, 81P45.

Key words and phrases. Operator entropies, JC-algebras, Operator inequalities, Noncommutative perspective. 
and more recently on quantum information theory. Subsequently, much effort were made to explicate and generalize these results. Notably, Effros [5] presented a simple approach to the operator inequalities of Lieb [12] by utilizing an operator version of perspective function. More specifically, let $f$ and $h$ be real continuous function on a closed interval $I \subset \mathbb{R}$ and $A$ and $B$ two positive commuting operators. Effros's perspective function was defined by

$$
(f \Delta h)(A, B)=f\left(\frac{A}{h(B)}\right) h(B) .
$$

Extending Effros [5], Ebadian and his collaborators [4 introduced a noncommutative perspective function $P_{f \triangle h}(A, B)$ of two variables $A$ and $B$ associated to $f$ and $h$, where $A$ is a self-adjoint operator and $B$ is a positive invertible operator on a Hilbert space $H$ with spectra in a closed interval $I$ containing 0 , by setting

$$
P_{f \triangle h}(A, B)=h(B)^{\frac{1}{2}} f\left(h(B)^{-\frac{1}{2}} A h(B)^{-\frac{1}{2}}\right) h(B)^{\frac{1}{2}}
$$

Note that in this approach the assumption of the commutativity of $A$ and $B$ is dropped and this should have a deep influence on quantum mechanics and quantum information theory. With such a powerful notion, Ebadian et al first proved the necessary and sufficient condition of jointly convexity for noncommutative generalized perspective function ibid., then they gave arguably the simplest proof of Lieb concavity theorem and Ando convexity theorem [19. More recently, in a series of papers [16, 20, 17, 18], Nikoufar investigated the convexity property of relative operator $(\alpha, \beta)$-entropy and obtained, later refined its lower and upper bounds by taking advantage of noncommutative perspective function approach.

In the present paper, we study the relative operator entropies in the more general settings of $\mathrm{C}^{*}$-algebras, real $\mathrm{C}^{*}$-algebras and JC-algebras. We show that all the operator inequalities on relative operator entropies still hold in our settings. Moreover, we refine the lower and upper bounds of the relative operator $(\alpha, \beta)$-entropy established by Nikoufar in [16] and [18] which improved the bounds for the relative operator entropy obtained earlier by Fujii and Kamei [8, 9].

In the rest of this section we give some background and fix the notation. The self-adjoint part $B(H)_{s a}$ of all bounded operators $B(H)$ on a complex Hilbert space $H$ is a Jordan algebra under the non-associative product $A \circ B:=\frac{1}{2}(A B+B A)$. A JC-algebra is a norm-closed Jordan subalgebra of the Jordan algebra $B(H)_{s a}$; it is an ordered space with order inherited from that of $B(H)_{s a}$ via the cone $B(H)_{+}$ of positive operators on $H$. The notion of JC-algebras arose in the work of Jordan, von Neumann, and Wigner on the axiomatic foundations of quantum mechanics. The 'observables' in a quantum system naturally constitute a JC-algebra and play the central role in the formulation of quantum theory. For $A, B$ in a unital JCalgebra, we have the important identity of Jordan algebras

$$
A B A=2(A \circ B) \circ A-A^{2} \circ B
$$

If $A$ is strictly positive, then for any $\alpha \in \mathbb{R}, A^{\alpha}$ is contained in JC-algebra generated by $A, 1$, which is automatically associative. More information on JC-algebras can be found in 22$]$

In this paper, $\mathcal{A}$ denotes a $\mathrm{C}^{*}$-algebra, or a real $\mathrm{C}^{*}$-algebra, or a JC-algebra, with a unit. For two strictly positive elements $A, B$ in $\mathcal{A}$ and $\alpha, \beta$ in $\mathbb{R}$, the operator 
$(\alpha, \beta)$-geometric mean is defined as

$$
A \#{ }_{(\alpha, \beta)} B=A^{\frac{\beta}{2}}\left(A^{-\frac{\beta}{2}} B A^{-\frac{\beta}{2}}\right)^{\alpha} A^{\frac{\beta}{2}}
$$

Note that if $\beta=1$, then $A \#_{(\alpha, 1)} B$ is the $\alpha$-geometric mean $A \#_{\alpha} B$ in the sense of Ando [3. The crucial observation is that relative operator entropies $S(A \mid B)$, $S_{\alpha}(A \mid B), S_{\alpha, \beta}(A \mid B)$ and perspective function $P_{f \triangle h}(A, B)$ defined in (1.1), (1.2), (1.3), (1.4) all lie in $\mathcal{A}$ for all three cases; in the case of a JC-algebra, this is due to (1.5) and functional calculus [1.

\section{Main Results}

For the special case of a $C^{*}$-algebra $\mathcal{A}=B(H)$, the following is Theorem 1 in 18.

Theorem 2.1. Let $\mathcal{A}$ be a $C^{*}$-algebra, or a real $C^{*}$-algebra, or a JC-algebra, with unit. Let $r, q, k$ and $h$ be real valued continuous functions on a closed interval $\mathbb{I}$ such that $h>0$ and $r(x) \leq q(x) \leq k(x)$. For a positive invertible element $A$ and a self-adjoint element $B$ in $\mathcal{A}$ such that the spectra of $A$ and $h(A)^{-1 / 2} B h(A)^{-1 / 2}$ are contained in $\mathbb{I}$,

$$
P_{r \triangle h}(B, A) \leq P_{q \triangle h}(B, A) \leq P_{k \triangle h}(B, A) .
$$

Proof. Let $f=q-r$ or $f=k-q$. Then $f\left(h(A)^{-\frac{1}{2}} B h(A)^{-\frac{1}{2}}\right) \geq 0$ since the three functional calculi are order preserving, cf. [6, 11, 1]. Therefore,

$$
h(A)^{\frac{1}{2}} f\left(h(A)^{-\frac{1}{2}} B h(A)^{-\frac{1}{2}}\right) h(A)^{\frac{1}{2}} \geq 0 .
$$

Remark 2.2. In general, the spectrum of an element in a real $C^{*}$-algebra is always symmetric with respect to the real axis. Therefore the three functional calculi for $C^{*}$-algebra, real $C^{*}$-algebra, and JC-algebra give rise to two different elements, $c f$. 6. 11, 1. For instance, for a normal element $A$ in a $C^{*}$-algebra or real $C^{*}$-algebra, and an appropriate continuous function $f, f(A)$ for the $C^{*}$-algebra is different for $f(A)$ for the real $C^{*}$-algebra since the spectra of $A$ in these two cases are different in general.

For self-adjoint element $A$ in a $C^{*}$-algebra, or real $C^{*}$-algebra, or $J C$-algebra, $f(A)$ gives the same element in all three cases.

The following is a quick application of Theorem 2.1 to three operator means, namely, weighted harmonic mean, weighted geometric mean, and weighted arithmetic mean in our settings, extending [2].

Proposition 2.3. Let $A, B$ be positive invertible elements in $\mathcal{A}$. For any $0 \leq \lambda \leq 1$,

$$
\left((1-\lambda) A^{-1}+\lambda B^{-1}\right)^{-1} \leq A^{\frac{1}{2}}\left(A^{-\frac{1}{2}} B A^{-\frac{1}{2}}\right)^{\lambda} A^{\frac{1}{2}} \leq(1-\lambda) A+\lambda B .
$$

Proof. One sees that for any $0 \leq \lambda \leq 1$

$$
\left((1-\lambda) 1+\lambda x^{-1}\right)^{-1} \leq x^{\lambda} \leq(1-\lambda) 1+\lambda x
$$

hold for all $x>0$. Applying Theorem 2.1 to the inequalities (2.3) with $h(x)=x$ gives (2.2). 
Remark 2.4. If $\lambda=\frac{1}{2}$, then the inequalities (2.2) become the classical ones for harmonic mean, geometric mean and arithmetic mean.

Suppose that $A, B$ are strictly positive elements in $\mathcal{A}$, which denotes a $\mathrm{C}^{*}$ algebra, or a real $\mathrm{C}^{*}$-algebra, or a JC-algebra, with a unit. With notation as in (1.3), for real numbers $\alpha \geq 0$ and $\beta>0$, we set

$$
\begin{aligned}
\mathrm{I} & =2 A^{\frac{\beta}{2}}\left[\left(1-2\left(1+A^{-\frac{\beta}{2}} B A^{-\frac{\beta}{2}}\right)^{-1}\right)\left(A^{-\frac{\beta}{2}} B A^{-\frac{\beta}{2}}\right)^{\alpha}\right] A^{\frac{\beta}{2}} \\
\mathrm{II} & =4 A \#_{(\alpha, \beta)} B-8 A^{\frac{\beta}{2}}\left[\left(A^{-\frac{\beta}{2}} B A^{-\frac{\beta}{2}}\right)^{\alpha}\left(\left(A^{-\frac{\beta}{2}} B A^{-\frac{\beta}{2}}\right)^{\frac{1}{2}}+1\right)^{-1}\right] A^{\frac{\beta}{2}} \\
\mathrm{III} & =A \#_{\left(\alpha+\frac{1}{2}, \beta\right)} B-A \#_{\left(\alpha-\frac{1}{2}, \beta\right)} B \\
\mathrm{~V} & =\frac{1}{2}\left(A \#_{(\alpha+1, \beta)} B-A \#_{(\alpha-1, \beta)} B\right)
\end{aligned}
$$

Then I, II, III, and V are in $\mathcal{A}$ by functional calculi in $\mathrm{C}^{*}$-algebra, real $\mathrm{C}^{*}$-algebra, and JC-algebra, 6, 11, 1].

Proposition 2.5. Let $A$ and $B$ be two positive invertible elements in $\mathcal{A}$. Then for $\alpha \geq 0$ and $\beta>0$,

(i) $A \#_{(\alpha, \beta)} B-A \#_{(\alpha-1, \beta)} B \leq \mathrm{I} \leq A \#_{(\alpha+1, \beta)} B-A \#_{(\alpha, \beta)} B$.

(ii) $A \#_{(\alpha, \beta)} B-A \#_{(\alpha-1, \beta)} B \leq \mathrm{V} \leq A \#_{(\alpha+1, \beta)} B-A \#_{(\alpha, \beta)} B$.

(iii) $A \#_{(0, \beta)} B-A \#_{(-1, \beta)} B \leq A \#_{(\alpha, \beta)} B-A \#_{(\alpha-1, \beta)} B$.

Proof. Here we only give the proof for the case of a unital JC-algebra; the proofs for other cases are similar and more straightforward.

Proof of (i). Let

$$
\begin{aligned}
& r(x)=x^{\alpha}-x^{\alpha-1}, \\
& q(x)=2\left(1-\frac{2}{x+1}\right) x^{\alpha}, \\
& k(x)=x^{\alpha+1}-x^{\alpha} .
\end{aligned}
$$

Then,

$$
r(x) \leq q(x) \leq k(x)
$$

for $x>0$ as shown in the proof of [18, Proposition 1].

Denoting $h(x)=x^{\beta}$, we have

$$
\begin{aligned}
& P_{r \triangle h}(B, A)=A \#_{(\alpha, \beta)} B-A \#_{(\alpha-1, \beta)} B \\
& P_{q \triangle h}(B, A)=2 A^{\frac{\beta}{2}}\left[\left(1-2\left(A^{-\frac{\beta}{2}} B A^{-\frac{\beta}{2}}+1\right)^{-1}\right)\left(A^{-\frac{\beta}{2}} B A^{-\frac{\beta}{2}}\right)^{\alpha}\right] A^{\frac{\beta}{2}}=\mathrm{I} \\
& P_{k \triangle h}(B, A)=A \#_{(\alpha+1, \beta)} B-A \#_{(\alpha, \beta)} B .
\end{aligned}
$$

One sees that $P_{r \triangle h}(B, A)$ and $P_{k \triangle h}(B, A)$ are in $\mathcal{A}$. Note also that

$$
\left[\left(1-2\left(A^{-\frac{\beta}{2}} B A^{-\frac{\beta}{2}}+1\right)^{-1}\right)\left(A^{-\frac{\beta}{2}} B A^{-\frac{\beta}{2}}\right)^{\alpha}\right]
$$

is an element in the associative JC-subalgebra generated by $A^{-\frac{\beta}{2}} B A^{-\frac{\beta}{2}}$ and 1 . Hence,

$$
2 A^{\frac{\beta}{2}}\left[\left(1-2\left(A^{-\frac{\beta}{2}} B A^{-\frac{\beta}{2}}+1\right)^{-1}\right)\left(A^{-\frac{\beta}{2}} B A^{-\frac{\beta}{2}}\right)^{\alpha}\right] A^{\frac{\beta}{2}} \in \mathcal{A} .
$$

Applying Theorem 2.1 to the inequalities (2.8), we obtain that

$$
A \#_{(\alpha, \beta)} B-A \#_{(\alpha-1, \beta)} B \leq \mathrm{I} \leq A \#_{(\alpha+1, \beta)} B-A \#_{(\alpha, \beta)} B,
$$


proving (i).

For (ii), the inequalities

$$
x^{\alpha}-x^{\alpha-1} \leq \frac{1}{2}\left(x^{\alpha+1}-x^{\alpha-1}\right) \leq x^{\alpha+1}-x^{\alpha}
$$

hold for all $x>0$, which can be found in the proof of [18, Proposition 1]. Using the perspective functions associated with the three functions in (2.9) with $h$ as in the proof of (i) and applying Theorem 2.1, (ii) follows.

(iii) Note that $1-\frac{1}{x} \leq x^{\alpha}-x^{\alpha-1}$ for all $x>0$. Applying Theorem 2.1 to this inequality with $h(x)=x^{\beta}$, we know (iii) is true.

It is well known that the following Hermite-Hadamard integral inequality, which motivated Choquet's theory, plays a significant role in the theory of convex functions, optimization theory and engineering [15].

Lemma 2.6. Let $f$ be a convex function on the interval $[a, b]$. Then,

$$
f\left(\frac{a+b}{2}\right) \leq \frac{1}{b-a} \int_{a}^{b} f(t) d t \leq \frac{f(a)+f(b)}{2} .
$$

Nowadays much attention is paid to generalize and extend this classical fundamental result for convex function. For example, El Farissi gives the following improved estimation in [7].

Lemma 2.7. Assume that $f:[a, b] \rightarrow \mathbb{R}$ is a convex function. Then we have

$$
f\left(\frac{a+b}{2}\right) \leq \sup _{\lambda \in[0,1]} l(\lambda) \leq \frac{1}{b-a} \int_{a}^{b} f(t) d t \leq \inf _{\lambda \in[0,1]} L(\lambda) \leq \frac{f(a)+f(b)}{2},
$$

where

$$
l(\lambda)=\lambda f\left(\frac{\lambda b+(2-\lambda) a}{2}\right)+(1-\lambda) f\left(\frac{(1+\lambda) b+(1-\lambda) a}{2}\right)
$$

and

$$
L(\lambda)=\frac{1}{2}[f(\lambda b+(1-\lambda) a)+\lambda f(a)+(1-\lambda) f(b)] .
$$

Theorem 2 in [18] states that for $\alpha \geq 0, \beta>0$, and positive invertible operators $A$ and $B$ in $B(H)$ with $A^{\beta} \leq B$,

$$
\mathrm{I} \leq S_{\alpha, \beta}(A \mid B) \leq \mathrm{V} .
$$

The following result refines (2.10) and extends it to our broader settings.

Theorem 2.8. Let $\alpha \geq 0, \beta>0$, and let $\mathcal{A}$, be a $C^{*}$-algebra or a real $C^{*}$-algebra or a JC-algebra, with a unit. If $A$ and $B$ are two positive invertible elements in $\mathcal{A}$ satisfying $A^{\beta} \leq B$, then

$$
\mathrm{I} \leq \mathrm{II} \leq S_{\alpha, \beta}(A \mid B) \leq \mathrm{III} \leq \mathrm{V} .
$$

Proof. For $\alpha \geq 0$ and $x \geq 1$, define $f(t)=\frac{x^{\alpha}}{t}-1,1 \leq t \leq x$. Then $l(\lambda)$ and $L(\lambda)$ defined in Lemma 2.7 are

$$
\begin{aligned}
& l(\lambda)=x^{\alpha} \frac{2 \lambda}{\lambda(x-1)+2}+x^{\alpha} \frac{2-2 \lambda}{\lambda(x-1)+(x+1)}-1 ; \\
& L(\lambda)=\frac{1}{2}\left(\frac{x^{\alpha}}{\lambda(x-1)+1}+\lambda x^{\alpha}+(1-\lambda) x^{\alpha-1}\right)-1 .
\end{aligned}
$$


Applying refined Hermite-Hadamard inequality (see Lemma 2.7) for $f$ on the interval $[1, x]$, we get

$$
f\left(\frac{x+1}{2}\right) \leq \sup _{\lambda \in[0,1]} l(\lambda) \leq \frac{1}{x-1} \int_{1}^{x}\left(\frac{x^{\alpha}}{t}-1\right) d t \leq \inf _{\lambda \in[0,1]} L(\lambda) \leq \frac{f(x)+f(1)}{2} .
$$

By extreme value theorem, it is easy to show that

$$
\begin{aligned}
& \sup _{\lambda \in[0,1]} l(\lambda)=l\left(\frac{1}{\sqrt{x}+1}\right)=\frac{4 x^{\alpha}}{(\sqrt{x}+1)^{2}}-1, \\
& \inf _{\lambda \in[0,1]} L(\lambda)=L\left(\frac{1}{\sqrt{x}+1}\right)=\frac{x^{\alpha}}{\sqrt{x}}-1 .
\end{aligned}
$$

And, a simple calculation shows that

$$
\begin{aligned}
f\left(\frac{x+1}{2}\right) & =\frac{2 x^{\alpha}}{x+1}-1 \\
\int_{1}^{x}\left(\frac{x^{\alpha}}{t}-1\right) d t & =x^{\alpha} \ln x-(x-1), \\
\frac{f(x)+f(1)}{2} & =\frac{1}{2}\left(x^{\alpha}+x^{\alpha-1}\right)-1 .
\end{aligned}
$$

Therefore,

$$
\begin{aligned}
\frac{2 x^{\alpha}}{x+1}-1 & \leq \frac{4 x^{\alpha}}{(\sqrt{x}+1)^{2}}-1 \leq \frac{1}{x-1}\left[x^{\alpha} \ln x-(x-1)\right] \\
& \leq \frac{x^{\alpha}}{\sqrt{x}}-1 \leq \frac{1}{2}\left(x^{\alpha}+x^{\alpha-1}\right)-1 .
\end{aligned}
$$

Let

$$
\begin{aligned}
& r(x)=2\left(1-\frac{2}{x+1}\right) x^{\alpha}, \\
& s(x)=4 x^{\alpha}-\frac{8 x^{\alpha}}{\sqrt{x}+1}, \\
& q(x)=x^{\alpha} \ln (x), \\
& j(x)=\frac{x^{\alpha}(x-1)}{\sqrt{x}}, \\
& k(x)=\frac{1}{2}\left(x^{\alpha+1}-x^{\alpha-1}\right) .
\end{aligned}
$$

Then

$$
r(x) \leq s(x) \leq q(x) \leq j(x) \leq k(x) .
$$

Denoting $h(t)=t^{\beta}$, we have

$$
\begin{aligned}
& P_{r \triangle h}(B, A)=2 A^{\frac{\beta}{2}}\left(1-2\left(1+A^{-\frac{\beta}{2}} B A^{-\frac{\beta}{2}}\right)^{-1}\right)\left(A^{-\frac{\beta}{2}} B A^{-\frac{\beta}{2}}\right)^{\alpha} A^{\frac{\beta}{2}}=\mathrm{I} \\
& P_{s \triangle h}(B, A)=4 A \#_{(\alpha, \beta)} B-8 A^{\frac{\beta}{2}}\left[\left(A^{-\frac{\beta}{2}} B A^{-\frac{\beta}{2}}\right)^{\alpha}\left(\left(A^{-\frac{\beta}{2}} B A^{-\frac{\beta}{2}}\right)^{\frac{1}{2}}+1\right)^{-1}\right] A^{\frac{\beta}{2}}=\mathrm{II} \\
& P_{q \triangle h}(B, A)=S_{\alpha, \beta}(A \mid B) \\
& P_{j \triangle h}(B, A)=A_{\left(\alpha+\frac{1}{2}, \beta\right)} B-A_{\left(\alpha-\frac{1}{2}, \beta\right)} B=\mathrm{III} \\
& P_{k \triangle h}(B, A)=\frac{1}{2}\left(A \#_{(\alpha+1, \beta)} B-A \#_{(\alpha-1, \beta)} B\right)=\mathrm{V} .
\end{aligned}
$$


Applying Theorem 2.1 to (2.12), we derive the inequality

$$
\mathrm{I} \leq \mathrm{II} \leq S_{\alpha, \beta}(A \mid B) \leq \mathrm{III} \leq \mathrm{V} .
$$

Coupling Theorem 2.8 with Proposition 2.5 we obtain

Corollary 2.9. If $A$ and $B$ are two positive invertible elements in $\mathcal{A}$, with $A^{\beta} \leq B$, and $\alpha \geq 0, \beta>0$, then

$$
\begin{aligned}
A \#_{(\alpha, \beta)} B-A \#_{(\alpha-1, \beta)} B & \leq \mathrm{I} \leq \mathrm{II} \\
& \leq S_{\alpha, \beta}(A \mid B) \\
& \leq \mathrm{III} \leq \mathrm{V} \\
& \leq A \#_{(\alpha+1, \beta)} B-A \#_{(\alpha, \beta)} B .
\end{aligned}
$$

Corollary 5 in [9] states that for positive invertible operators $A$ and $B$ in $B(H)$,

$$
A-A B^{-1} A \leq S(A \mid B) \leq B-A .
$$

Under the assumption $A \leq B, 18$ provides the following sharper result for the relative operator entropy:

$$
\begin{aligned}
A-A B^{-1} A & \leq 2\left(A-2 A(A+B)^{-1} A\right) \leq S(A \mid B) \\
& \leq \frac{1}{2}\left(B-A B^{-1} A\right) \leq B-A .
\end{aligned}
$$

The following result refines (2.13), (2.14), and extends them to our settings.

Corollary 2.10. If $A$ and $B$ are two positive invertible elements in $\mathcal{A}$, with $A \leq B$, then

$$
\begin{aligned}
A-A B^{-1} A & \leq 2\left(A-2 A(A+B)^{-1} A\right) \\
& \leq 4 A-8 A\left(A \#_{\frac{1}{2}} B+A\right)^{-1} A \\
& \leq S(A \mid B) \\
& \leq A \#_{\frac{1}{2}} B-A \#_{-\frac{1}{2}} B \\
& \leq \frac{1}{2}\left(B-A B^{-1} A\right) \\
& \leq B-A .
\end{aligned}
$$

Theorem 2.11. If $A$ and $B$ are two positive invertible elements in $\mathcal{A}$ with $A^{\beta} \geq B$, and $\alpha \geq 0, \beta>0$, then

$$
\mathrm{V} \leq \mathrm{III} \leq S_{\alpha, \beta}(A \mid B) \leq \mathrm{II} \leq \mathrm{I}
$$

Proof. For $\alpha \geq 0$ and $0<x \leq 1$, define $f(t)=\frac{x^{\alpha}}{t}-1, t \in[x, 1]$. Then $l(\lambda)$ and $L(\lambda)$ defined in Lemma 2.7 are

$$
\begin{aligned}
l(\lambda) & =x^{\alpha} \frac{2 \lambda}{\lambda(1-x)+2 x}+x^{\alpha} \frac{2(1-\lambda)}{\lambda(1-x)+(x+1)}-1 \\
L(\lambda) & =\frac{1}{2}\left(\frac{x^{\alpha}}{\lambda(1-x)+x}+\lambda x^{\alpha-1}+(1-\lambda) x^{\alpha}\right)-1 .
\end{aligned}
$$


By applying refined Hermite-Hadamard inequality (see Lemma 2.7) for $f$ on the interval $[x, 1]$, we get

$$
f\left(\frac{x+1}{2}\right) \leq \sup _{\lambda \in[0,1]} l(\lambda) \leq \frac{1}{1-x} \int_{x}^{1}\left(\frac{x^{\alpha}}{t}-1\right) d t \leq \inf _{\lambda \in[0,1]} L(\lambda) \leq \frac{f(x)+f(1)}{2} .
$$

By extreme value theorem, we know that

$$
\begin{aligned}
& \sup _{\lambda \in[0,1]} l(\lambda)=l\left(\frac{\sqrt{x}}{\sqrt{x}+1}\right)=\frac{4 x^{\alpha}}{(\sqrt{x}+1)^{2}}-1, \\
& \inf _{\lambda \in[0,1]} L(\lambda)=L\left(\frac{\sqrt{x}}{\sqrt{x}+1}\right)=\frac{x^{\alpha}}{\sqrt{x}}-1 .
\end{aligned}
$$

Thus,

$$
\begin{aligned}
\frac{2 x^{\alpha}}{x+1}-1 & \leq \frac{4 x^{\alpha}}{(\sqrt{x}+1)^{2}}-1 \leq \frac{1}{x-1}\left[x^{\alpha} \ln x-(x-1)\right] \\
& \leq \frac{x^{\alpha}}{\sqrt{x}}-1 \leq \frac{1}{2}\left(x^{\alpha}+x^{\alpha-1}\right)-1 .
\end{aligned}
$$

Let

$$
\begin{aligned}
& r(x)=2\left(1-\frac{2}{x+1}\right) x^{\alpha}, \\
& s(x)=4 x^{\alpha}-\frac{8 x^{\alpha}}{\sqrt{x}+1}, \\
& q(x)=x^{\alpha} \ln (x), \\
& j(x)=\frac{x^{\alpha}(x-1)}{\sqrt{x}}, \\
& k(x)=\frac{1}{2}\left(x^{\alpha+1}-x^{\alpha-1}\right) .
\end{aligned}
$$

Then

$$
k(x) \leq j(x) \leq q(x) \leq s(x) \leq r(x) .
$$

Applying Theorem 2.1 to (2.16), the desired inequalities follow.

Similarly, combing Theorem 2.11 with Proposition 2.5, we have

Corollary 2.12. If $A$ and $B$ are two positive invertible elements in $\mathcal{A}$, with $A^{\beta} \geq$ $B$, and $\alpha \geq 0, \beta>0$, then

$$
\begin{aligned}
A \#_{(\alpha, \beta)} B-A \#_{(\alpha-1, \beta)} B & \leq \mathrm{V} \leq \mathrm{III} \\
& \leq S_{\alpha, \beta}(A \mid B) \\
& \leq \mathrm{II} \leq \mathrm{I} \\
& \leq A \#_{(\alpha+1, \beta)} B-A \#_{(\alpha, \beta)} B .
\end{aligned}
$$

The following improves Corollary 5 in [9] and extends it to our more general settings. 
Corollary 2.13. If $A$ and $B$ are two positive invertible elements in $\mathcal{A}$, with $A \geq B$, then

$$
\begin{aligned}
A-A B^{-1} A & \leq \frac{1}{2}\left(B-A B^{-1} A\right) \\
& \leq A \#_{\frac{1}{2}} B-A \#_{-\frac{1}{2}} B \\
& \leq S(A \mid B) \\
& \leq 4 A-8 A\left(A \#_{\frac{1}{2}} B+A\right)^{-1} A \\
& \leq 2\left(A-2 A(A+B)^{-1} A\right) \\
& \leq B-A .
\end{aligned}
$$

\section{Further Refinements}

Suppose that $A, B$ are two positive invertible elements. For any real number $\delta>0, \alpha \geq 0$ and $\beta>0$, we denote

$$
\begin{aligned}
\mathrm{I}^{\prime} & =(\ln \delta+2) A \#_{(\alpha, \beta)} B-2 \delta A^{\frac{\beta}{2}}\left[\left(A^{-\frac{\beta}{2}} B A^{-\frac{\beta}{2}}+\delta\right)^{-1}\left(A^{-\frac{\beta}{2}} B A^{-\frac{\beta}{2}}\right)^{\alpha}\right] A^{\frac{\beta}{2}} \\
\mathrm{II}^{\prime} & =(\ln \delta+4) A \#_{(\alpha, \beta)} B-8 \sqrt{\delta} A^{\frac{\beta}{2}}\left[\left(\left(A^{-\frac{\beta}{2}} B A^{-\frac{\beta}{2}}\right)^{\frac{1}{2}}+\sqrt{\delta}\right)^{-1}\left(A^{-\frac{\beta}{2}} B A^{-\frac{\beta}{2}}\right)^{\alpha}\right] A^{\frac{\beta}{2}} \\
\mathrm{III}^{\prime} & =\left(\frac{1}{\sqrt{\delta}} A \#_{\left(\alpha+\frac{1}{2}, \beta\right)} B-\sqrt{\delta} A_{\left(\alpha-\frac{1}{2}, \beta\right)} B\right)+\ln \delta \#_{(\alpha, \beta)} B \\
\mathrm{~V}^{\prime} & =\frac{1}{2}\left(\frac{1}{\delta} A \#_{(\alpha+1, \beta)} B-\delta A \#_{(\alpha-1, \beta)} B\right)+\ln \delta A \#_{(\alpha, \beta)} B .
\end{aligned}
$$

Theorem 3.1. Let $A$ and $B$ be two positive invertible elements in $\mathcal{A}$ which is a $C^{*}$-algebra or real $C^{*}$ or $J C$-algebra $\mathcal{A}$, with unit, satisfying $\delta \geq 1, \delta A^{\beta} \leq B$, and $\alpha \geq 0$ and $\beta \geq 0$. Then

$$
\mathrm{I}^{\prime} \leq \mathrm{II}^{\prime} \leq S_{\alpha, \beta}(A \mid B) \leq \mathrm{III}^{\prime} \leq \mathrm{V}^{\prime}
$$

For $\delta \leq 1$ and $B \leq \delta A^{\beta}$, the reversed inequalities hold.

Proof. Let $x \geq \delta$. Then $x / \delta \geq 1$. By substituting $x$ with $x / \delta$, the following inequalities hold:

(a) $\left(\ln \delta+2\left(1-\frac{2 \delta}{x+\delta}\right)\right) x^{\alpha} \leq\left(\ln \delta+4-\frac{8 \sqrt{\delta}}{\sqrt{x}+\sqrt{\delta}}\right) x^{\alpha} \leq x^{\alpha} \ln \alpha$.

(b) $x^{\alpha} \ln \alpha \leq x^{\alpha+\frac{1}{2}} \frac{1}{\sqrt{\delta}}-x^{\alpha-\frac{1}{2}} \sqrt{\delta}+x^{\alpha} \ln \delta \leq \frac{x^{\alpha+1}}{2 \delta}-\frac{x^{\alpha-1}}{2} \delta+x^{\alpha} \ln \delta$.

Let

$$
\begin{aligned}
& r(x)=\left[\ln \delta+2\left(1-\frac{2 \delta}{x+\delta}\right)\right] x^{\alpha}, \\
& s(x)=\left[\ln \delta+4-\frac{8 \sqrt{\delta}}{\sqrt{x}+\sqrt{\delta}}\right] x^{\alpha}, \\
& q(x)=x^{\alpha} \ln (x), \\
& j(x)=x^{\alpha+\frac{1}{2}} \frac{1}{\sqrt{\delta}}-x^{\alpha-\frac{1}{2}} \sqrt{\delta}+x^{\alpha} \ln \delta, \\
& k(x)=\frac{x^{\alpha+1}}{2 \delta}-\frac{x^{\alpha-1}}{2} \delta+x^{\alpha} \ln \delta, \\
& h(t)=t^{\beta} .
\end{aligned}
$$


Then

$$
\begin{aligned}
& P_{r \triangle h}(B, A)=(\ln \delta+2) A \#_{(\alpha, \beta)} B-2 \delta A^{\frac{\beta}{2}}\left[\left(A^{-\frac{\beta}{2}} B A^{-\frac{\beta}{2}}+\delta\right)^{-1}\left(A^{-\frac{\beta}{2}} B A^{-\frac{\beta}{2}}\right)^{\alpha}\right] A^{\frac{\beta}{2}}=\mathrm{I}^{\prime} \\
& P_{s \triangle h}(B, A)=(\ln \delta+4) A \#_{(\alpha, \beta)} B-8 \sqrt{\delta} A^{\frac{\beta}{2}}\left[\left(\left(A^{-\frac{\beta}{2}} B A^{-\frac{\beta}{2}}\right)^{\frac{1}{2}}+\sqrt{\delta}\right)^{-1}\left(A^{-\frac{\beta}{2}} B A^{-\frac{\beta}{2}}\right)^{\alpha}\right] A^{\frac{\beta}{2}}=\mathrm{II}^{\prime} \\
& P_{s \triangle h}(B, A)=S_{\alpha, \beta}(A \mid B) \\
& P_{j \triangle h}(B, A)=\left(\frac{1}{\sqrt{\delta}} A \#_{\left(\alpha+\frac{1}{2}, \beta\right)} B-\sqrt{\delta} A \#_{\left(\alpha-\frac{1}{2}, \beta\right)} B\right)+\ln \delta A \#_{(\alpha, \beta)} B=\mathrm{III}^{\prime} \\
& P_{k \triangle h}(B, A)=\frac{1}{2}\left(\frac{1}{\delta} A \#_{(\alpha+1, \beta)} B-\delta \#_{(\alpha-1, \beta)} B\right)+\ln \delta A \#_{(\alpha, \beta)} B=\mathrm{V}^{\prime} .
\end{aligned}
$$

The results follow immediately by applying Theorem 2.1

Theorem 3.1 above refines Theorem 3 in [18] and extends it to our broader settings, where it is shown

$$
\mathrm{I}^{\prime} \leq S_{\alpha, \beta}(A \mid B) \leq \mathrm{V}^{\prime} .
$$

Proposition 3.2. For $\alpha \geq 0, \beta>0, \delta \geq 1$, and positive invertible elements $A$ and $B$ in $\mathcal{A}$ with $\delta A^{\beta} \leq B$, we have

$$
\mathrm{II} \leq \mathrm{II}^{\prime} \quad \text { and } \quad \mathrm{III}^{\prime} \leq \mathrm{III},
$$

where II and III are defined in (2.5) and (2.6) respectively. For $\delta \leq 1$ and $B \leq \delta A^{\beta}$ the reversed inequalities hold.

Proof. (i) Proof of II $\leq \mathrm{II}^{\prime}$. Denote $\Gamma(t)=\left(\ln t+4-\frac{8 \sqrt{t}}{\sqrt{x}+\sqrt{t}}\right) x^{\alpha}$ and $x \geq \delta \geq$ $t \geq 1$. Then

$$
\Gamma^{\prime}(t)=\frac{\left(x^{\frac{1}{2}}-t^{\frac{1}{2}}\right)^{2}}{\left(x^{\frac{1}{2}}+t^{\frac{1}{2}}\right)^{2} t} \geq 0
$$

Suppose $x$ is fixed, then $\Gamma(1) \leq \Gamma(\delta)$, that is,

$$
\left(4-\frac{8}{\sqrt{x}+1}\right) x^{\alpha} \leq\left(\ln t+4-\frac{8 \sqrt{t}}{\sqrt{x}+\sqrt{t}}\right) x^{\alpha}
$$

Let $h(t)=t^{\beta}$. By applying Theorem 2.1 to the inequality (3.2), the result follows directly.

(ii) Proof of III' $\leq$ III. Let $\Omega(t)=x^{\alpha+\frac{1}{2}} \frac{1}{\sqrt{t}}-x^{\alpha-\frac{1}{2}} \sqrt{t}+x^{\alpha} \ln t$ with $x \geq \delta \geq t \geq 1$. Then

$$
\Omega^{\prime}(t)=-\frac{x^{\alpha}}{2 t^{\frac{3}{2}}}\left(x^{\frac{1}{4}}-x^{-\frac{1}{4}} t^{\frac{1}{2}}\right)^{2} \leq 0 .
$$

Suppose $x$ is fixed. Then $\Omega(\delta) \leq \Omega(1)$, which is equivalent to

$$
x^{\alpha+\frac{1}{2}} \frac{1}{\sqrt{\delta}}-x^{\alpha-\frac{1}{2}} \sqrt{\delta}+x^{\alpha} \ln \delta \leq x^{\alpha+\frac{1}{2}}-x^{\alpha-\frac{1}{2}} .
$$

Let $h(t)=t^{\beta}$. By applying Theorem 2.1 to the inequality (3.3), the result follows immediately.

Combining Theorem 3.1 and Proposition 3.2, we have

Corollary 3.3. Let $A$ and $B$ be two positive invertible elements in $\mathcal{A}$ which is a $C^{*}$-algebra or real $C^{*}$-algebra or JC-algebra, with a unit, let $\alpha \geq 0, \beta>0$, and let $\mathrm{I}$ and $\mathrm{V}$ be as in (2.4) and (2.7) respectively. 
(i) If $\delta \geq 1$ and $\delta A^{\beta} \leq B$, then

$$
\mathrm{I} \leq \mathrm{II} \leq \mathrm{II}^{\prime} \leq S_{\alpha, \beta}(A \mid B) \leq \mathrm{III}^{\prime} \leq \mathrm{V}^{\prime} \leq \mathrm{V} .
$$

(ii) If $\delta \leq 1$ and $\delta A^{\beta} \geq B$, then

$$
\mathrm{V} \leq \mathrm{V}^{\prime} \leq \mathrm{III}^{\prime} \leq S_{\alpha, \beta}(A \mid B) \leq \mathrm{II}^{\prime} \leq \mathrm{II} \leq \mathrm{I}
$$

The following corollary provides a sharper refinement in our settings for the lower and upper bounds of the relative operator entropy established by Nikoufar in [16, 18, which improved the bounds obtained earlier by Fujii and Kamei [8, 9 .

Corollary 3.4. Let $A$ and $B$ be two positive invertible elements in $\mathcal{A}$ which is a $C^{*}$-algebra or real $C^{*}$-algebra or JC-algebra, with a unit.

(i) If $\delta \geq 1$ and $\delta A \leq B$, then

$$
\begin{aligned}
A-A B^{-1} A & \leq 2\left[A-2 A(A+B)^{-1} A\right] \\
& \leq(\ln \delta) A+2\left[A-2 \delta A(\delta A+B)^{-1} A\right] \\
& \leq(\ln \delta+4) A-8 \sqrt{\delta} A\left(A \#_{\frac{1}{2}} B+\sqrt{\delta} A\right)^{-1} A \\
& \leq S(A \mid B) \\
& \leq\left(\frac{1}{\sqrt{\delta}} A \#_{\frac{1}{2}} B-\sqrt{\delta} A \#_{-\frac{1}{2}} B\right)+(\ln \delta) A \\
& \leq \frac{1}{2}\left(\frac{1}{\delta} B-\delta A B^{-1} A\right)+(\ln \delta) A \\
& \leq \frac{1}{2}\left(B-A B^{-1} A\right) \\
& \leq B-A .
\end{aligned}
$$

(ii) If $\delta \leq 1$ and $\delta A \geq B$, then

$$
\begin{aligned}
A-A B^{-1} A & \leq \frac{1}{2}\left(B-A B^{-1} A\right) \\
& \leq \frac{1}{2}\left(\frac{1}{\delta} B-\delta A B^{-1} A\right)+(\ln \delta) A \\
& \leq\left(\frac{1}{\sqrt{\delta}} A \#_{\frac{1}{2}} B-\sqrt{\delta} A \#_{-\frac{1}{2}} B\right)+(\ln \delta) A \\
& \leq S(A \mid B) \\
& \leq(\ln \delta+4) A-8 \sqrt{\delta} A\left(A \#_{\frac{1}{2}} B+\sqrt{\delta} A\right)^{-1} A \\
& \leq(\ln \delta) A+2\left[A-2 \delta A(\delta A+B)^{-1} A\right] \\
& \leq 2\left[A-2 A(A+B)^{-1} A\right] \\
& \leq B-A .
\end{aligned}
$$

\section{REFERENCES}

[1] E. M. Alfsen and F. W. Shultz. Geometry of state spaces of operator algebras. Mathematics: theory \& applications. Birkhäuser, 2003.

[2] T. Ando. Topics on operator inequalities. Division of Applied Mathematics, Research Institute of Applied Electricity, Hokkaido University, 1978.

[3] T. Ando. Concavity of certain maps on positive definite matrices and applications to Hadamard products. Linear Algebra and its Applications, 26:203-241, 1979. 
[4] A. Ebadian, I. Nikoufar, and M. E. Gordji. Perspectives of matrix convex functions. Proceedings of the National Academy of Sciences, 108(18):7313-7314, 2011.

[5] E. G. Effros. A matrix convexity approach to some celebrated quantum inequalities. Proceedings of the National Academy of Sciences, 106(4):1006-1008, 2009.

[6] S. Eilers, D. Olesen, and G. K. Pedersen, editors. C*-Algebras and Their Automorphism Groups. Pure and Applied Mathematics. Academic Press, Second Edition edition, 2018.

[7] A. El Farissi. Simple proof and refinement of Hermite-Hadamard inequality. J. Math. Ineq, $4(3): 365-369,2010$.

[8] J. I. Fujii and E. Kamei. Relative operator entropy in noncommutative information theory. Math. Jap., 34:341-348, 1989.

[9] J. I. Fujii and E. Kamei. Uhlmann's interpolational method for operator means. Math. Jap., 34:541-547, 1989.

[10] T. Furuta. Parametric extensions of Shannon inequality and its reverse one in Hilbert space operators. Linear algebra and its applications, 381:219-235, 2004.

[11] B. Li. Real Operator Algebras. World Scientific, 2003.

[12] E. H. Lieb. Convex trace functions and the Wigner-Yanase-Dyson conjecture. Advances in Mathematics, 11(3):267 - 288, 1973.

[13] E. H. Lieb and M. B. Ruskai. Proof of the strong subadditivity of quantum-mechanical entropy. Journal of Mathematical Physics, 14(12):1938-1941, 1973.

[14] M. Nakamura and H. Umegaki. A note on the entropy for operator algebras. Proc. Japan Acad., 37(3):149-154, 1961.

[15] C. P. Niculescu and L.-E. Persson. Convex Functions and Their Applications: A Contemporary Approach. Springer, 2018.

[16] I. Nikoufar. On operator inequalities of some relative operator entropies. Advances in Mathematics, 259:376 - 383, 2014.

[17] I. Nikoufar. Convexity of parameter extensions of some relative operator entropies with a perspective approach. Glasgow Mathematical Journal, page 1-8, 2019.

[18] I. Nikoufar. Improved operator inequalities of some relative operative entropies. Positivity, 24(1):241-251, 2020.

[19] I. Nikoufar, A. Ebadian, and M. Eshaghi Gordji. The simplest proof of Lieb concavity theorem. Advances in Mathematics, 248:531 - 533, 2013.

[20] I. Nikoufar and M. Shamohammadi. Generalized perspectives of functions of several variables. Mathematical Communications, 23(1):1-8, 2018.

[21] I. E. Segal. A note on the concept of entropy. Journal of Mathematics and Mechanics, 9(4):623-629, 1960.

[22] D. Topping. Jordan Algebras of Self-Adjoint Operators. Mem. Amer. Math. Soc. American Mathematical Society, 1965.

[23] H. Umegaki. Conditional expectation in an operator algebra. iv. entropy and information. Kodai Math. Sem. Rep., 14(2):59-85, 1962.

Department of Mathematics, University of Georgia, Athens, GA, 30602

Email address: szwang@uga.edu

Department of Mathematics, University of Georgia, Athens, GA, 30602

Email address: ezrawang@uga.edu 